Convectons in periodic and bounded domains

This article has been downloaded from IOPscience. Please scroll down to see the full text article.

2010 Fluid Dyn. Res. 42025505

(http://iopscience.iop.org/1873-7005/42/2/025505)

The Table of Contents and more related content is available

Download details:

IP Address: 147.83.27.47

The article was downloaded on 09/02/2010 at $16: 33$

Please note that terms and conditions apply. 


\title{
Convectons in periodic and bounded domains
}

\author{
Isabel Mercader $^{1}$, Oriol Batiste ${ }^{1}$, Arantxa Alonso ${ }^{1}$ and Edgar Knobloch ${ }^{2}$ \\ ${ }^{1}$ Departament de Física Aplicada, Universitat Politècnica de Catalunya, Barcelona, Spain \\ ${ }^{2}$ Department of Physics, University of California, Berkeley, CA 94720, USA
}

Received 24 July 2009

Published 21 December 2009

Online at stacks.iop.org/FDR/42/025505

Communicated by A Gilbert

\begin{abstract}
Numerical continuation is used to compute spatially localized convection in a binary fluid with no-slip laterally insulating boundary conditions and the results are compared with the corresponding ones for periodic boundary conditions (PBC). The change in the boundary conditions produces a dramatic change in the snaking bifurcation diagram that describes the organization of localized states with PBC: the snaking branches turn continuously into a large amplitude state that resembles periodic convection with defects at the sidewalls. Odd parity convectons are more affected by the boundary conditions since the sidewalls suppress the horizontal pumping action that accompanies these states in spatially periodic domains.
\end{abstract}

(Some figures in this article are in colour only in the electronic version)

\section{Introduction}

Many fluid systems exhibit spatially localized structures in both two (Ghorayeb and Mojtabi 1997, Blanchflower 1999, Batiste et al 2005, 2006, Dawes 2007, Assemat et al 2008, Bergeon and Knobloch 2008) and three (Blanchflower and Weiss 2002, Mercader et al 2008) dimensions. Of these the localized structures or convectons arising in binary fluid convection are perhaps the best studied.

A binary mixture with negative separation ratio heated from below develops a stabilizing concentration gradient via the (anomalous) Soret effect, resulting in the presence of subcritical steady convection (figure 1(a)). This subcritical regime favors the presence of convectons. These come in two types, even and odd, and are present in an interval of Rayleigh numbers called the pinning region (Pomeau 1986) containing multiple convectons of different lengths and either parity (Batiste et al 2006). In horizontally unbounded domains these localized structures appear simultaneously with the (subcritical) primary branch of spatially periodic steady convection. The resulting convectons are spatially extended at small amplitude, but become strongly localized when followed numerically to larger amplitude by decreasing the 

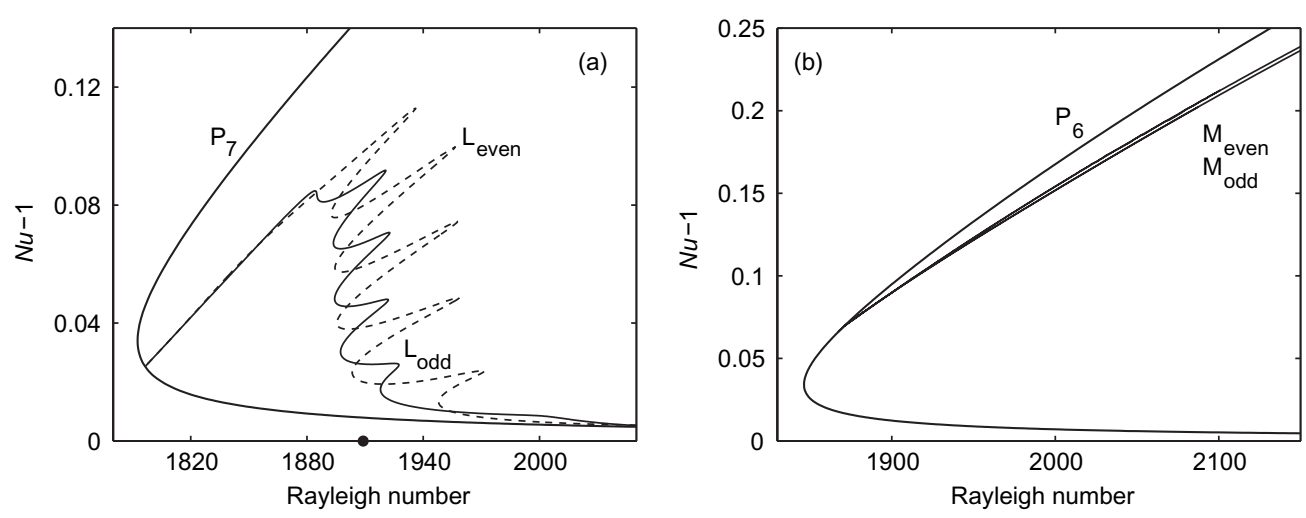

Figure 1. (a) Branches of stationary spatially periodic $\left(\mathrm{P}_{7}\right)$ and stationary spatially localized states of even ( $\mathrm{L}_{\text {even }}$, dashed line) and odd ( $\mathrm{L}_{\text {odd }}$, solid line) parity in a $\Gamma=14$ periodic domain showing the normalized convective Nusselt number $N u-1 \equiv \Gamma^{-1} \int_{-\Gamma / 2}^{\Gamma / 2} \partial_{z} \theta(x, z=1) \mathrm{d} x$ as a function of the Rayleigh number $R$. The pinning region of the odd convectons is narrower than that of the even convectons. Both convecton branches terminate together on $\mathrm{P}_{7}$. (b) The same but showing the branch $\mathrm{P}_{6}$ together with a pair of mixed modes $\mathrm{M}_{\mathrm{even}}, \mathrm{M}_{\mathrm{odd}}$ of opposite parity that bifurcate from $\mathrm{P}_{6}$ above its saddle-node. The conduction state loses stability at a Hopf bifurcation (solid dot) but only time-independent states are shown

Rayleigh number. Once their amplitude and length are comparable with the amplitude and wavelength of steady spatially periodic convection on the upper branch (figure 1(a)) both convecton branches enter the pinning region and begin to snake back and forth across it (figure 1(a)) as the convectons grow in length by nucleating additional convection rolls at both ends. With periodic boundary conditions (PBC) in the horizontal this process continues until the length of the convecton becomes comparable with the available spatial domain when the convecton branches turn over towards the saddle-node of the periodic branch and leave the pinning region (Assemat et al 2008, Bergeon et al 2008, Bergeon and Knobloch 2008). We show here that this picture changes fundamentally in the presence of physically realistic (i.e. nonperiodic) lateral boundary conditions.

The snaking behavior shown in figure 1(a) is a consequence of the formation of a heteroclinic cycle between the conduction state and periodic convection, i.e. a solution profile that connects the conduction state at $x=-\infty$ to the periodic state, with a second connection from the periodic state back to the conduction state as $x \rightarrow \infty$. In generic systems, the formation of this type of orbit is a higher codimension phenomenon but in systems that are reversible in space the return connection follows from symmetry and the heteroclinic cycle may become generic or structurally stable. Numerical computations suggest that this is the case for binary fluid convection: the pinning or snaking interval is filled with heteroclinic connections between the conduction and periodic states. The two boundaries of this region correspond to tangencies between the unstable (stable) manifold of the conduction state and the center-stable (center-unstable) manifold of the periodic state, respectively (Woods and Champneys 1999, Coullet et al 2000, Beck et al 2009).

In this paper, we study snaking behavior in binary fluid convection in the presence of both PBC and physically realistic lateral boundaries (Mercader et al 2009) and compare the two. The Boussinesq equations describing the system are nondimensionalized using the layer depth as the unit of length and the thermal diffusion time in the vertical as the unit of time, 
and take the dimensionless form (Batiste et al 2006)

$$
\begin{aligned}
& \mathbf{u}_{t}+(\mathbf{u} \cdot \nabla) \mathbf{u}=-\nabla P+\sigma R[(1+S) \theta-S \eta] \hat{\mathbf{z}}+\sigma \nabla^{2} \mathbf{u}, \\
& \theta_{t}+(\mathbf{u} \cdot \nabla) \theta=w+\nabla^{2} \theta \\
& \eta_{t}+(\mathbf{u} \cdot \nabla) \eta=\tau \nabla^{2} \eta+\nabla^{2} \theta
\end{aligned}
$$

together with the incompressibility condition $\nabla \cdot \mathbf{u}=0$. Here $\mathbf{u} \equiv(u, w)$ is the velocity field in $(x, z)$ coordinates, $P$ is the pressure and $\theta$ denotes the departure of the temperature from its conduction profile, in units of the imposed temperature difference $\Delta T$. The variable $\eta$ is defined such that its gradient represents the dimensionless flux of the larger molecular weight component. Thus $\eta \equiv \theta-\Sigma$, where $C \equiv 1-z+\Sigma$ is the concentration of this component in units of the concentration difference that develops across the layer via the Soret effect in response to the imposed $\Delta T$. The system is specified by four dimensionless parameters: the Rayleigh number $R$ that provides a dimensionless measure of $\Delta T$, the separation ratio $S$ that measures the concentration contribution to the buoyancy force due to the Soret effect, and the Prandtl and Lewis numbers $\sigma$ and $\tau$. The equations are solved in the domain $-\Gamma / 2 \leqslant x \leqslant \Gamma / 2,0 \leqslant z \leqslant 1$, with boundary conditions $\mathbf{u}=\theta=\eta_{z}=0$ on $z=0,1$, and either PBC in $x$ with period $\Gamma$, or insulating closed container boundary conditions (ICCBC) $\mathbf{u}=\theta_{x}=\eta_{x}=0$ on $x= \pm \Gamma / 2$. The ICCBC used here differ from the CCBC employed by Mercader et al (2009), which assumed perfectly conducting lateral walls. Throughout we use the (experimental) parameter values $\sigma=7, \tau=0.01$ and $S=-0.1$. In the following, we focus on time-independent solutions only, but note that the primary instability of the conduction state as $R$ increases is a Hopf bifurcation. This bifurcation takes place at $R=1909$ and is indicated in figure 1(a) by a solid dot. In small periodic domains, this bifurcation leads to (typically unstable) branches of traveling and standing waves, but in larger domains a spatiotemporally chaotic state called dispersive chaos is found for supercritical Rayleigh numbers and small to moderate values of $|S|$ (Alonso et al 2007); the time-independent convectons discussed here emerge from this state as described by Batiste et al (2006).

With PBC the above equations have the symmetry $O(2) \times Z_{2}$, where $R_{x} \in O(2)$ acts by $(u(x, z), w(x, z), \theta(x, z), \eta(x, z)) \rightarrow(-u(-x, z), w(-x, z), \theta(-x, z), \eta(-x, z))$ relative to a suitable origin, and $R_{z} \in Z_{2}$ acts by $(u(x, z), w(x, z), \theta(x, z), \eta(x, z)) \rightarrow(u(x, 1-z)$, $-w(x, 1-z),-\theta(x, 1-z),-\eta(x, 1-z))$; the continuous part of the symmetry $O(2)$ corresponds to translations in $x$. The presence of this symmetry is important, and is responsible, for example, for the bifurcation of a circle of periodic states from the conduction state at the primary bifurcation.

When the boundary conditions are changed to ICCBC (or CCBC as in Mercader et al (2009)) the translation invariance is broken and the symmetry that remains is the symmetry $Z_{2} \times Z_{2} \sim D_{2}$ generated by $R_{x}$ and $R_{z}$. In this case, we expect solutions that are invariant under $R_{x}$, i.e. satisfying $(u(x, z), w(x, z), \theta(x, z), \eta(x, z))=(-u(-x, z)$, $w(-x, z), \theta(-x, z), \eta(-x, z))$, as well as solutions that are invariant under $R_{x} \circ R_{z}$, i.e. satisfying $(u(x, z), w(x, z), \theta(x, z), \eta(x, z))=-(u(-x, 1-z), w(-x, 1-z), \theta(-x, 1-z)$, $\eta(-x, 1-z))$. In the following, we refer to the former as even and the latter as odd, in both cases with respect to the line $x=0$. These are the only steady solutions that can bifurcate from the conduction state. Both can be computed by imposing appropriate symmetries on the solutions. Solutions invariant under $R_{z}$ do not bifurcate at finite Rayleigh number $R$. 
(a)

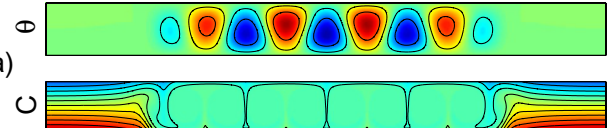

(b)
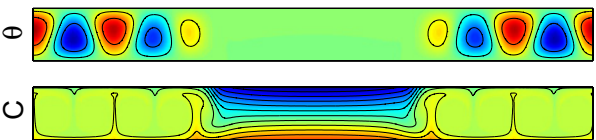

(c)

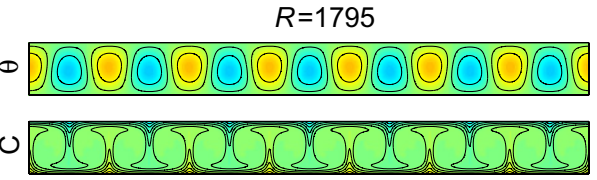

(d)

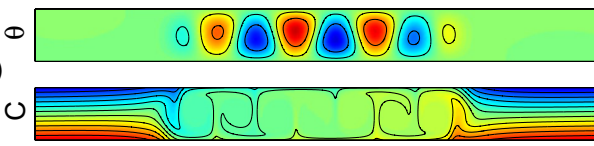

$R=1919$

(e)

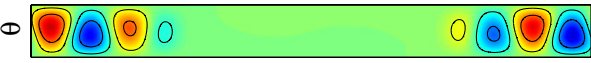

(f)

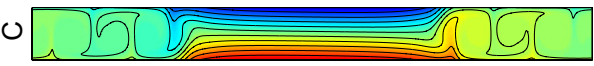

$R=1795$

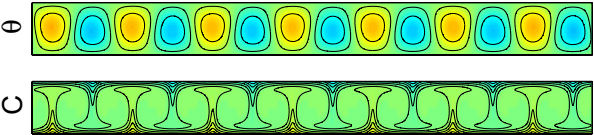

Figure 2. (a) An even parity convecton and (b) an even parity hole, both of which bifurcate together from the same $\mathrm{P}_{7}$ state (c) at $R=1795$. (d) An odd parity convecton and (e) an odd parity hole, both of which also bifurcate together at $R=1795$ but from a different $\mathrm{P}_{7}$ state (f), related to (c) by translation by $\lambda / 4$. All calculations use a $\Gamma=14$ periodic domain. The states are visualized in terms of contours of constant temperature fluctuation $\theta$ (upper panels) and contours of constant concentration $C$ (lower panels). Here and elsewhere all solution profiles use the same color table to indicate the amplitude of the temperature and concentration fields. The average concentration is $C=1 / 2$ (green color).

\section{Periodic boundary conditions}

Figure 1(a) shows the result of numerical branch following in a periodic domain of length $\Gamma=14$. The branches of even and odd convectons (labeled $\mathrm{L}_{\text {even }}$ and $\mathrm{L}_{\text {odd }}$ ) are shown in dashed and solid lines, respectively. Both branches terminate together on the branch $\mathrm{P}_{7}$ of stationary periodic states with $n=7$ pairs of rolls, just below the saddle-node on the latter, but the pinning region for the odd convectons is noticeably narrower than the corresponding region for even convectons. This is a consequence of the horizontal pumping of concentration associated with odd parity convectons (Batiste et al 2006, Mercader et al 2009).

Figure 2(a) shows an even parity convecton at $R=1950$, i.e. inside the pinning region. Other even parity states can be produced from this state by translation by $\Gamma / 2$ and/or reflection in the horizontal midplane, i.e. by applying the operation $R_{z}$. Two of the resulting even states represent convectons located in the center of the domain, while two represent states with a hole in the center. Of these four states the two states shown in figures 2(a) and (b) possess upflows at the same location as the periodic state $\mathrm{P}_{7}$, with wavelength $\lambda=\Gamma / 7$, shown in figure 2(c). Thus these three states are phase matched and this is the reason why the states in figures 2(a) and (b), shown at $R=1950$, bifurcate together from the state in figure 2(c) at $R=1795$. The remaining two even parity states are phase matched with a $\lambda / 2$ translate of the periodic state, and hence also bifurcate together. Since these two even parity periodic states are related by translation this bifurcation also takes place at $R=1795$. For the odd parity states the same construction leads to four odd parity states. Of these the two shown in figures 2(d) and (e), for $R=1919$, are phase matched with the odd parity periodic state shown in figure 2(f). Consequently these two states also bifurcate together, and since the state in figure $2(\mathrm{f})$ is related to the state in figure 2 (c) by translation by $\lambda / 4$ this bifurcation also takes place at $R=1795$. The same argument shows, finally, that the remaining two odd states bifurcate together from the $3 \lambda / 4$ translate of the state shown in figure $2(\mathrm{c})$, and do so at $R=1795$ 

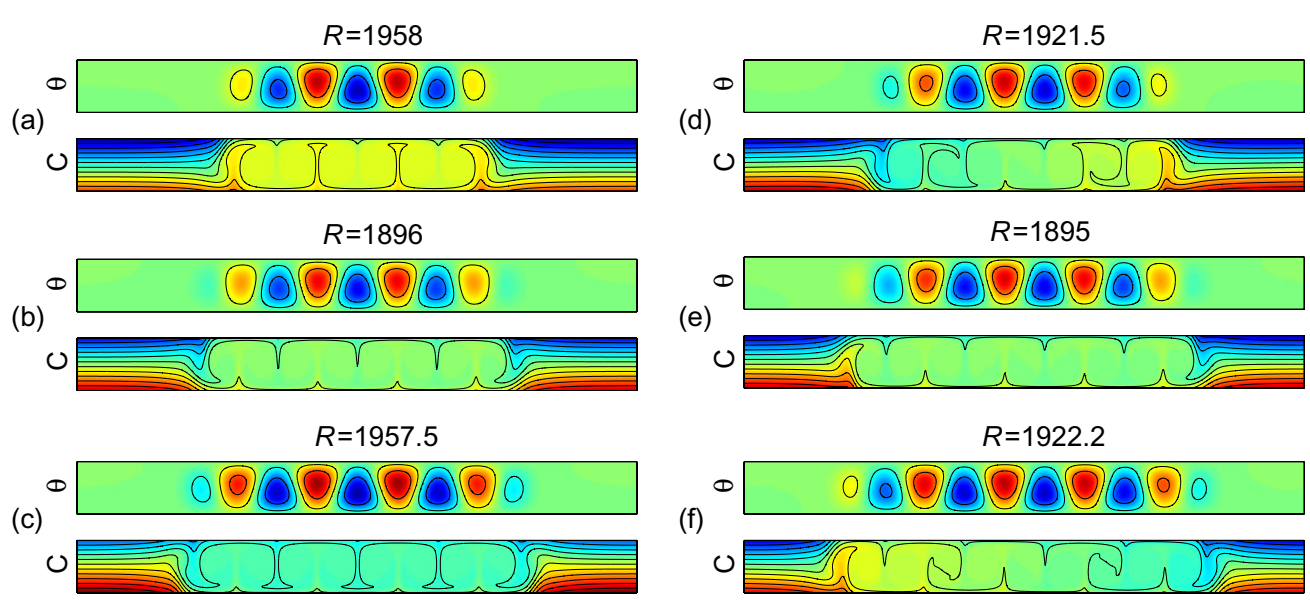

Figure 3. (a)-(c) Even parity convectons at successive saddle-nodes on the $\mathrm{L}_{\text {even }}$ branch. States (a) and (c) are on the right of the pinning region, while (b) is on the left. (d)-(f) Odd parity convectons at successive saddle-nodes on the $\mathrm{L}_{\text {odd }}$ branch. States (d) and (f) are on the right of the pinning region, whereas (e) is on the left. The states on the right side of the pinning region are characterized by strong vortices at either end of the structure and hence strong entrainment; those on the left side of the pinning region have weak vortices in the front regions, and very little concentration is entrained. Thus, the convectons on the left side of the pinning region are characterized by homogenized concentration that is equal to the average concentration.

as well. This completes the discussion of the termination of the snaking branches of even and odd convectons. Note that from this perspective there are in fact eight snaking branches, four corresponding to snaking convectons and four to snaking holes, although there are only two distinct snaking branches in bifurcation diagrams such as that shown in figure 1(a). If we identify symmetry-related branches the secondary bifurcation in figure 1(a) is a pitchfork from a group orbit of periodic states, despite the fact that the odd and even parity branches are unrelated by symmetry. This is a consequence of weak spatial resonance between the periodic state and the bifurcating state (here a 1:7 resonance) (Prat et al 1998, Bergeon et al 2008).

We remark that in figure 2(a) the vortices at either end of the convecton entrain lighter fluid from above while the hole is embedded between vortices that entrain heavier fluid from below (figure 2(b)). In contrast, in the odd parity convecton shown in figure 2(d) the vortices at the two ends of the convecton rotate in the same sense (counterclockwise). As a result the vortex at the right entrains heavier fluid from the bottom while that at the left entrains lighter fluid from the top, setting up a visible mean concentration gradient across the convecton. The situation is reversed for the hole state (figure 2(e)).

Figure 3 shows even and odd convectons at successive saddle-nodes on $\mathrm{L}_{\text {even }}$ and $\mathrm{L}_{\mathrm{odd}}$, respectively. The figure reveals that the end vortices repeatedly change direction as one proceeds from saddle-node to saddle-node along the right boundary of the pinning region, as additional vortices are nucleated pairwise at the edges of each convecton. As a result the convectons at the right boundary are characterized by strong entrainment of concentration, leading either to a change in the mean concentration within the convecton (even parity convectons) or a concentration gradient within the convecton (odd parity convectons). In contrast, the saddle-nodes along the left boundary correspond to transitions between these states, and for these the end vortices are very weak, with almost no entrainment taking place (figure 3). 
$R=2100$

(a)

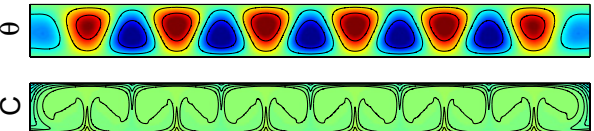

$R=2100$

(b)

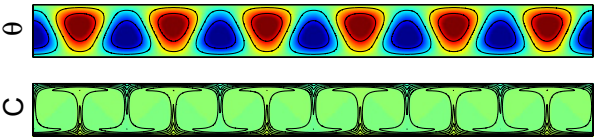

(c)

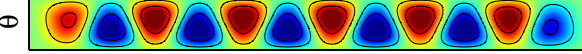

(d)

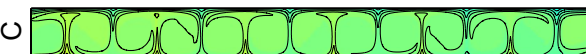

$R=2100$

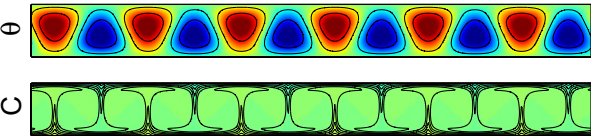

Figure 4. (a) An even parity mixed mode $M_{\text {even }}$. (b) The phase-matched even parity representative of the $\mathrm{P}_{6}$ state. (c) An odd parity mixed mode $\mathrm{M}_{\text {odd }}$. (d) The phase-matched odd parity representative of the $\mathrm{P}_{6}$ state. Hole states are omitted. All solutions are computed at $R=2100$ in a $\Gamma=14$ periodic domain.

In figure 1(b) we show the $\mathrm{P}_{6}$ branch when $\Gamma=14$. This branch consists of six pairs of rolls within the period $\Gamma$, and behaves in much the same way as branch $\mathrm{P}_{7}$ (figure $1(\mathrm{a})$ ). This branch also exhibits a secondary bifurcation to opposite parity states. This time these correspond to mixed modes. These resemble the periodic state but have a defect somewhere within the wavetrain, which we may choose to place on the boundary of the periodic domain or in the center in order to construct states with well-defined parity relative to $x=0$. This secondary bifurcation is also of Eckhaus-type (Bergeon et al 2008) and so has the same properties as the bifurcation terminating the snaking branches already discussed. Thus, there are eight branches, four of even parity states and four of odd parity states, and these bifurcate pairwise from phase-matched representatives from the $\mathrm{P}_{6}$ branch. Figure 4(a) shows an even parity state with defects at the domain boundary, whereas figure 4(b) shows the phase-matched even parity periodic $\mathrm{P}_{6}$ state at the same value of the Rayleigh number $R$. Figure 4(c) shows an odd parity defect state at the same $R$, while figure 4(d) shows the phase-matched odd parity periodic $\mathrm{P}_{6}$ state, also at this $R$. However, this time instead of snaking the bifurcating branches all extend monotonically to large amplitude. That this is so should not come as a surprise. As explained by Bergeon et al (2008) there are in general only two distinct snaking branches (of single pulse states), and these are typically associated with the primary pattern branch only.

The corresponding results for $\Gamma=13$ are shown in figures 5(a) and (b). This time the bifurcation diagrams are completely different. The odd convectons still terminate on $\mathrm{P}_{7}$ while the even convectons now terminate on $\mathrm{P}_{6}$. This possibility, predicted recently in the context of the Swift-Hohenberg equation (Bergeon et al 2008, Dawes 2009), has also been seen in natural doubly diffusive convection (Bergeon et al 2008). Our discussion of secondary bifurcations on the $\mathrm{P}_{7}$ and $\mathrm{P}_{6}$ branches applies exactly as before, and indicates that each bifurcation produces eight states, four with even parity and four with odd parity. This time, however, the odd parity states bifurcating from $\mathrm{P}_{7}$ snake, while the even states do not, and vice versa for the $\mathrm{P}_{6}$ branch. Once again only two branches snake while the remaining ones correspond to mixed modes. At large amplitude these resemble the mixed modes shown in figures 4(a) and (c). Note that despite terminating on a different branch the pinning region for odd convectons remains substantially narrower than that for even convectons.

\section{Closed container boundary conditions}

Figures 6(a) and (b) show the corresponding results in a $\Gamma=14$ domain when ICCBC are imposed at $x= \pm \Gamma / 2$ and compare the results with those of the corresponding PBC case. As discussed by Mercader et al (2009) the traditional snaking diagram is greatly perturbed by this 

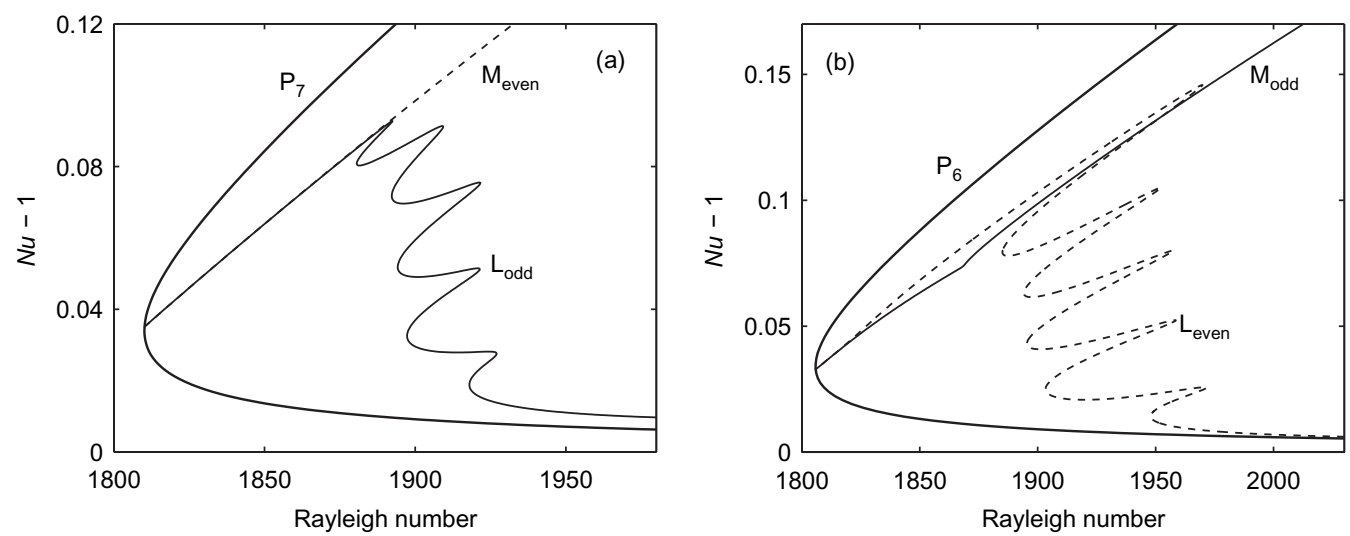

Figure 5. Branches of spatially periodic states $P_{6}$ and $P_{7}$ together with branches of spatially localized states of odd $\left(\mathrm{L}_{\text {odd }}\right)$ parity and even $\left(\mathrm{L}_{\text {even }}\right)$ parity in a $\Gamma=13$ periodic domain showing the convective Nusselt number $N u-1$ as a function of the Rayleigh number $R$. (a) The odd convectons $\left(\mathrm{L}_{\mathrm{odd}}\right)$ terminate on $\mathrm{P}_{7}$ together with a monotonic branch of even mixed modes $\left(\mathrm{M}_{\mathrm{even}}\right)$. (b) The even convectons $\left(\mathrm{L}_{\mathrm{even}}\right)$ terminate on $P_{6}$ together with a monotonic branch of odd mixed modes $\left(\mathrm{M}_{\mathrm{odd}}\right)$.
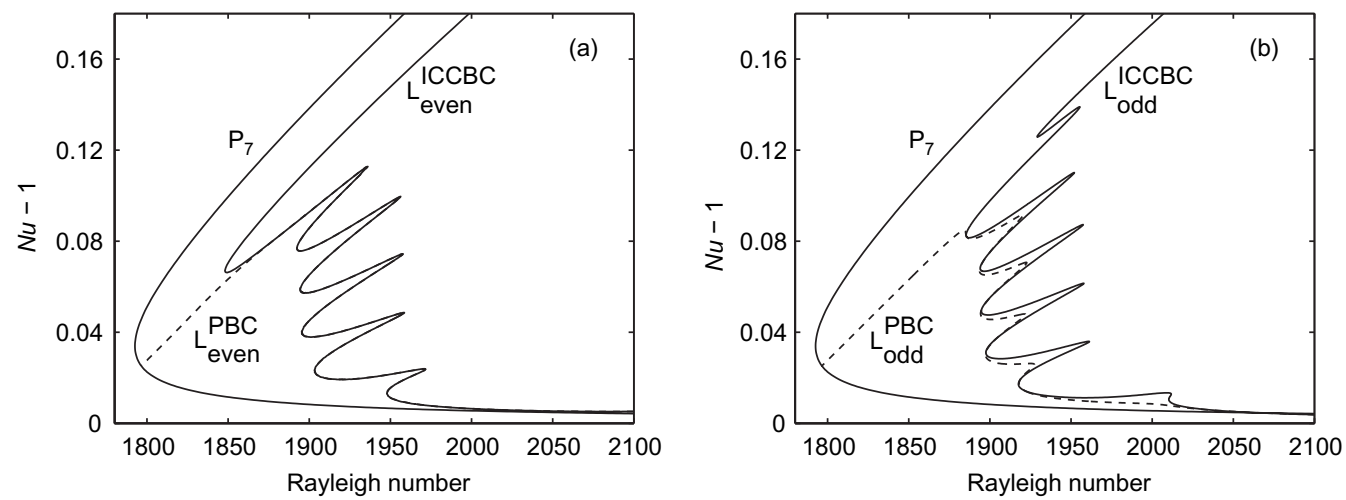

Figure 6. Comparison of the bifurcation diagrams in a $\Gamma=14$ domain with PBC and ICCBC. (a) Even parity localized states; the two branches coincide until the domain is almost filled. (b) Odd parity localized states; the two branches differ throughout as a consequence of the suppression of horizontal pumping by odd convectons in the presence of ICCBC.

type of boundary condition. Instead of coexistence between snaking and periodic branches the snaking branches now turn continuously into large amplitude states that take the place of the competing periodic states and resemble the mixed mode states computed using PBC. This is so for both even (figure 6(a)) and odd (figure 6(b)) parity states. Localized states computed with ICCBC at successive saddle-nodes are shown in figure 7. In addition the ICCBC increase substantially the width of the pinning region for the odd parity state and hence exert an effect on odd parity convectons even when the convectons are localized well away from the walls. Indeed the width of the pinning region of the odd parity states is now identical to that of the even parity states.

Figure 6(a) shows that until the domain fills with convection the even parity convecton branches with ICCBC and PBC track one another very closely, indicating that the pinning of the fronts to the structured state between them that is responsible for the snaking behavior of 

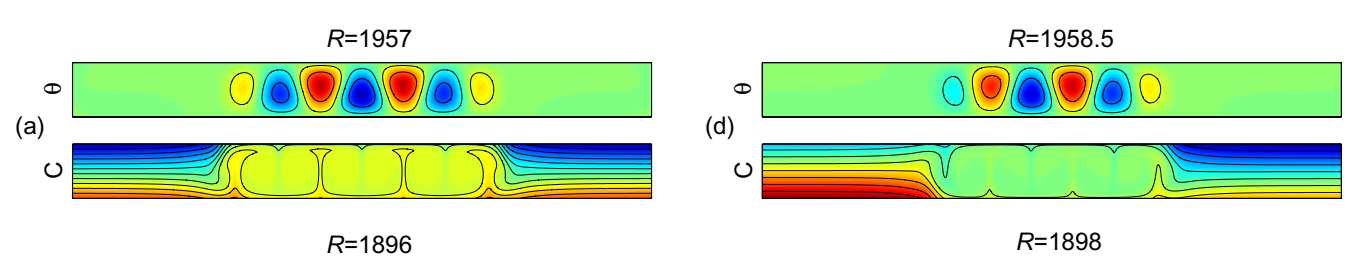

(b)
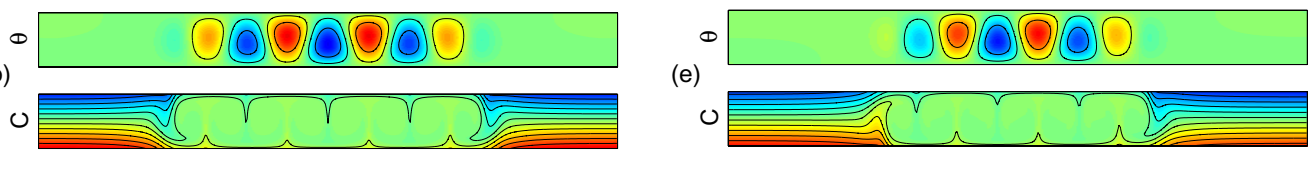

$R=1957$

(c)
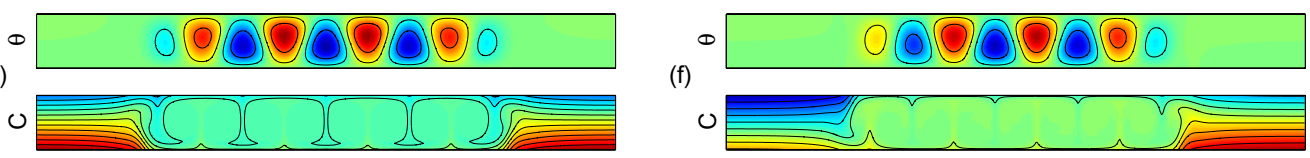

Figure 7. (a)-(c) Even parity convectons with ICCBC in a $\Gamma=14$ container at successive saddlenodes on the $\mathrm{L}_{\text {even }}$ branch. States (a) and (c) are on the right of the pinning region, while (b) is on the left. (d)-(f) Odd parity convectons with ICCBC in a $\Gamma=14$ container at successive saddlenodes on the $\mathrm{L}_{\text {odd }}$ branch. States (d) and (f) are on the right of the pinning region, while (e) is on the left. The states on the right of the pinning region are characterized by strong vortices at either end of the structure and hence strong entrainment; those on the left of the pinning region have weak vortices in the front regions, and very little concentration is entrained.

the convecton branches, is unaffected by the lateral walls whenever the convecton has even parity. Figure 6(b) shows that this is not the case for odd parity convectons, suggesting that the horizontal pumping of concentration by odd parity convectons is responsible (Mercader et al 2009). However, the snaking behavior with ICCBC still resembles the PBC case qualitatively, and indeed the left boundaries of the two pinning regions coincide (see below).

As explained by Mercader et al (2009) odd parity convectons in the presence of closed container boundary conditions should be thought of as being one half of a two-convecton state filling a domain of length $2 \Gamma$ obtained by reflecting the odd convecton in $x= \pm \Gamma / 2$. Such a reflection yields exact solutions in the case of periodic or Neumann boundary conditions and results in a state in which the pumping effects of oppositely oriented odd convectons cancel out, producing a more or less spatially uniform concentration (and temperature) in the void region between them (figures $7(\mathrm{~d})$ and (f)), and eliminating the mean concentration gradient present in odd convectons computed with PBC (figures 3(d) and (f)). The concentration level on either side of the convecton depends on whether the convectons pump toward one another or away from one another, and in a domain of period $2 \Gamma$ the resulting concentration jump balances the pumping action of the individual convectons. It is for this reason that the twoconvecton state with equidistant convectons matches, at least approximately, the solutions found here with insulating no-slip boundary conditions at $x= \pm \Gamma / 2$.

To confirm this scenario we examine the flux balance in the fore and aft regions of the odd parity convecton shown in figure $7(\mathrm{~d})$. We denote the midplane concentration to the left of the convecton by $C^{-}$and to the right by $C^{+}$; the mean midplane concentration inside the convecton is $\bar{C}$. We suppose that the convecton pumps concentration from right to left with flux $F$. Then flux balance at the left front of the convecton, required for the existence of a steady state, leads to the estimate $F \approx \tau\left(C^{-}-\bar{C}\right) / \delta$, while that at the right front leads to $F \approx \tau\left(\bar{C}-C^{+}\right) / \delta$. Here $\delta$ is the width of the front region, assumed to be the same fore and aft; since the midplane temperature is nearly uniform across the domain no Soret terms appear. 

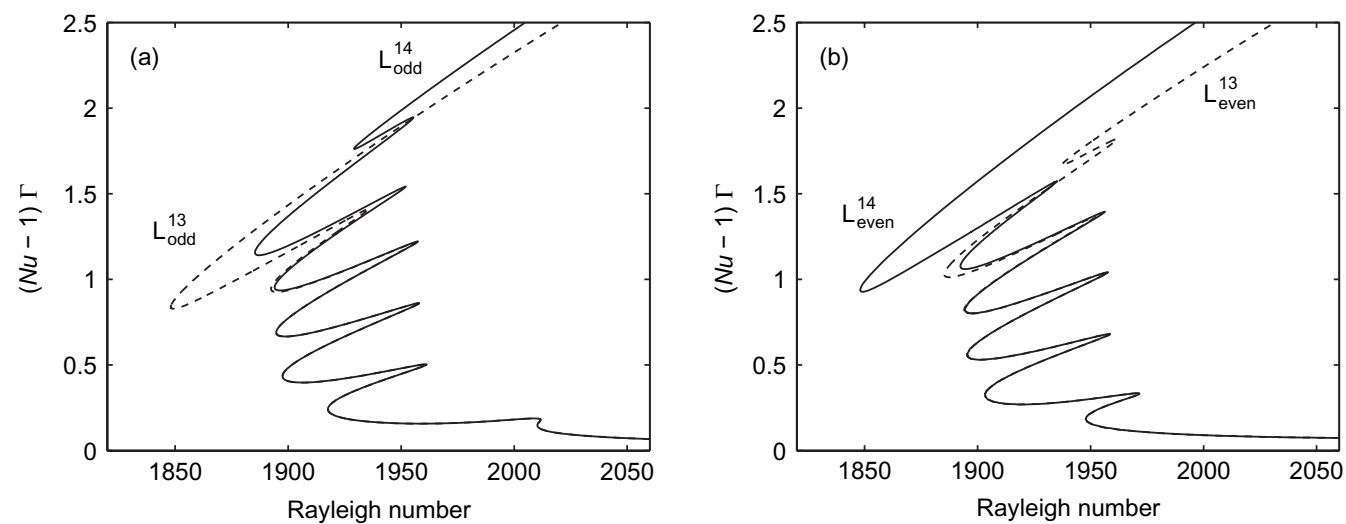

Figure 8. Comparison of (a) odd convecton branches and (b) even convecton branches in $\Gamma=13$ and $\Gamma=14$ domains with ICCBC. The branches coincide until the domain is almost full.

It follows that $\bar{C} \approx\left(C^{+}+C^{-}\right) / 2$, a conclusion that agrees very well with the computations shown in figures $7(\mathrm{~d})$ and (f). The same argument applied to odd or even convectons at a left saddle-node leads to the prediction $C^{-}=\bar{C}=C^{+}$since $F=0$ (no entrainment by end vortices). This is so for both PBC (figure 3) and ICCBC (figure 7) since the boundary conditions exert little influence when $F=0$, in agreement with the computations reported in figures 3(b) and (e) and 7(b) and (e). This prediction explains, moreover, why the left boundary of the pinning region for odd convectons is unaffected by the boundary conditions (figure 6(b)) - and hence coincides with the left boundary of the even convecton snaking region-while the right boundary is affected dramatically. Finally, the above argument also explains the depletion of the concentration outside the even convecton in figure 7(a) and its enhancement outside the even convecton in figure 7(c).

Figure 8 compares the results for both odd and even convectons in $\Gamma=13$ and $\Gamma=14$ domains with ICCBC. Both odd convecton branches and both even convecton branches track each other until the domain is almost full and its precise length begins to be felt. The fact that odd convectons in a $\Gamma=13$ domain behave like even convectons in a $\Gamma=14$ domain and vice versa is a consequence of the fact that the wavelength of the periodic state is $\lambda \approx 2$. Thus with ICCBC the width of the snaking regions is independent of both parity and domain length, in contrast to the $\mathrm{PBC}$ case.

\section{Summary}

The dramatic change from the bifurcation diagram in figure 1(a) to that in figures 6(a) and (b) is a consequence of changing the boundary conditions from PBC to ICCBC and is reproduced by the Swift-Hohenberg equation with non-Neumann boundary conditions (Houghton and Knobloch 2009, Kozyreff et al 2009). In both systems the behavior observed in figures 6(a) and (b) arises as a consequence of the destruction of the spatially periodic state by the boundary conditions appropriate for closed containers. In both systems the remaining mixed modes take over the role of the large amplitude periodic state, and the snaking branches gradually turn into the mixed modes with increasing amplitude. This behavior, referred to by Mercader et al (2009) as snaking without bistability, is robust with respect to changes in $\Gamma$. 


\section{Acknowledgments}

This work was supported in part by the National Science Foundation under grant DMS0605238, DGICYT under grant FIS2006-08954 and by AGAUR under grant 2005SGR-0024.

\section{References}

Alonso A, Batiste O, Meseguer A and Mercader I 2007 Complex dynamical states in binary mixture convection with weak negative Soret coupling Phys. Rev. E 75026310

Assemat P, Bergeon A and Knobloch E 2008 Spatially localized states in Marangoni convection in binary mixtures Fluid Dyn. Res. 40 852-76

Batiste O and Knobloch E 2005 Simulations of localized states of stationary convection in ${ }^{3} \mathrm{He}-{ }^{4} \mathrm{He}$ mixtures Phys. Rev. Lett. 95244501

Batiste O, Knobloch E, Alonso A and Mercader I 2006 Spatially localized binary fluid convection J. Fluid Mech. 560 149-58

Beck M, Knobloch J, Lloyd D J B, Sandstede B and Wagenknecht T 2009 Snakes, ladders and isolas of localized patterns SIAM J. Math. Anal. 41 936-72

Bergeon A, Burke J, Knobloch E and Mercader I 2008 Eckhaus instability and homoclinic snaking Phys. Rev. E 78 046201

Bergeon A and Knobloch E 2008 Spatially localized states in natural doubly diffusive convection Phys. Fluids 20 034102

Blanchflower S 1999 Magnetohydrodynamic convectons Phys. Lett. A 261 74-81

Blanchflower S and Weiss N O 2002 Three-dimensional magnetohydrodynamic convectons Phys. Lett. A 294 297-303

Coullet P, Riera C and Tresser C 2000 Stable static localized structures in one dimension Phys. Rev. Lett. 84 3069-72

Dawes J H P 2007 Localized convection cells in the presence of a vertical magnetic field J. Fluid Mech. 570 $385-406$

Dawes J H P 2009 Modulated and localized states in a finite domain SIAM J. Appl. Dyn. Syst. 8909-30

Ghorayeb K and Mojtabi A 1997 Double diffusive convection in a vertical rectangular cavity Phys. Fluids $92339-48$

Houghton S M and Knobloch E 2009 Homoclinic snaking in bounded domains Phys. Rev. E 80026210

Kozyreff G, Assemat P and Chapman S J 2009 Influence of boundaries on localized patterns Phys. Rev. Lett. 103164501

Mercader I, Alonso A and Batiste O 2008 Spatiotemporal dynamics near the onset of convection for binary mixtures in cylindrical containers Phys. Rev. E 77036313

Mercader I, Batiste O, Alonso A and Knobloch E 2009 Localized pinning states in closed containers: homoclinic snaking without bistability Phys. Rev. E 80025201

Pomeau Y 1986 Front motion, metastability and subcritical bifurcations in hydrodynamics Physica D 23 3-11

Prat J, Mercader I and Knobloch E 1998 Resonant mode interactions in Rayleigh-Bénard convection Phys. Rev. E 58 3145-56

Woods P D and Champneys A R 1999 Heteroclinic tangles and homoclinic snaking in an unfolding of a degenerate Hamiltonian-Hopf bifurcation Physica D 129 147-70 